\title{
Editorial
}

\section{Resisting the current to understand the tides}

\author{
Journal of Direct, Data and Digital Marketing Practice (2014) 16, 1-2. doi:10.1057/dddmp.2014.40
}

One of the challenges of editing a journal that is only published quarterly is ensuring that its contents are relevant to readers. There are two risks: one, being seduced by what is current, that is, something that exercises an immediate pull, but only briefly; and two, missing an emerging trend that does warrant closer scrutiny. In the digital world - most especially social media — these risks are high. Combined with the Journal's production schedule is the cycle followed by our academic contributors. For them, a new trend or technique needs to be identified, a theoretical framework drawn up and checked against existing literature, a research project commissioned and completed, before a whitepaper can be written. In most cases, that will take a minimum of a year. Add in the review process that all such submissions to the Journal undergo and it will typically be 18 months at a minimum from first idea to final, published article.

So it is always gratifying to be publishing content that chimes with live, ongoing activities. Take 'The ups and downs of online dating' by Dr Jiyao Xun (see pp. 51-60). It sets out to prove that users of online dating websites do so in a state of heightened emotion that affects what they do - something that researchers had previously ignored. Just as the editor set to work on the paper, a real-world example burst into the headlines. US-based dating website OK Cupid revealed that it had been carrying out experiments on its users to understand how different early experiences affect dating outcomes. Most notably, it revealed that it had spoofed results from its own matching algorithm, flipping them around to present well-matched partners with ill-matched ones while telling them they were ideally suited. The result? Exactly the same as when individuals are genuinely matched by the site's algorithm. In other words, setting up an expectation influences behaviour, exactly as Dr Xun proves.

Yet more real-world relevance can be found across our other papers. 'The value of Twitter for sports fans' by Jo Williams, Susan J. Chinn and James Suleiman (pp. 36-50) coincides with the transfer market and restart of the Premier League season here in the United Kingdom. Fans will have been lighting up their Twitter feeds with the latest news and excitement about their team's resumption of play. No coincidence, surely, that all of that chatter about transfers happens to coincide with the push for season ticket renewals - one of the most important revenue streams for sports clubs.

It may seem as if this plethora of digital channels could become overwhelming for consumers, leading to delayed choices or purchases. But in 'Clicking through choice' (pp. 24-35), Steven C. Huff and Aaron C. Johnson prove empirically that expanding the number of options on offer to consumers can paradoxically lead to them making more choices - a hugely valuable finding for digital marketers in their constant search for optimization.

Marketers themselves may be forgiven for feeling overwhelmed by the sheer variety of options confronting them. Whether conventional marketers or digital natives, the channels that need to be considered in the marketing mix have exploded in number, even as the resources available to operate within them have contracted. That has left many feeling uncertain and even disempowered. One solution is to take on training in the new specialist areas, something that Merlin Stone looks at in depth in his opinion piece, 'Building digital skills through training' (pp. 3-14).

As Stone points out, there has never been a greater need, more demand or depth of provision for digital marketing training. Yet many of the options available are not fit for purpose. One reason is the rate of evolution within digital marketing - by the time a course has been developed, the market will have moved on and some of the channels may not even exist. But equally, as Stone points out, too many have 


\section{桨. Editorial}

fallen prey to the lure of the current, focusing excessively on the latest techniques to have emerged especially in social media - with no consideration of their value to the business and its marketers. That is not a complaint that can be levelled at the courses offered by the IDM itself. Its history in reflecting the constantly changing environment for marketers, combined with its academic rigour, means that IDM training continues to lead the field. It is not something the Institute takes lightly or as a given, but it is something that members can rely on.

David Reed FIDM 